\title{
Synthesis of MgO Nanoparticles Using Artemisia abrotanum Herba Extract and Their Antioxidant and Photocatalytic Properties
}

\author{
Renata Dobrucka ${ }^{1}$
}

Received: 6 May 2016/Accepted: 3 August 2016/Published online: 2 September 2016

(c) The Author(s) 2016. This article is published with open access at Springerlink.com

\begin{abstract}
The synthesis of metal oxide nanoparticles with the use of plant extract is a promising alternative to traditional chemical methods. The aim of this work was to fabricate $\mathrm{MgO}$ nanoparticles using the Artemisia abrotanum herb water extract. The biologically synthesized $\mathrm{MgO}$ nanoparticles were characterized by UV-Visible spectroscopy, Fourier transform infrared spectroscopy (FTIR), X-ray diffraction (XRD), scanning electron microscopy (SEM) with EDS profile and transmission electron microscopy (TEM). XRD studies confirmed that pure monoclinic crystallite structures of $\mathrm{MgO}$ nanoparticles were formed. The average size of $\mathrm{MgO}$ nanoparticles was found to be $10 \mathrm{~nm}$. EDS profile confirmed the signal characteristic of magnesium and oxygen. FTIR analysis confirmed the presence of active compounds responsible for the stabilization of $\mathrm{MgO}$ nanoparticles. The synthesized nanoparticles showed good catalytic activity in the reduction of methyl orange (MO). $\mathrm{MgO}$ nanoparticles also exhibit very good antioxidant properties.
\end{abstract}

Keywords Magnesium oxide nanoparticles ·

Biosynthesis · Catalytic activity

\section{Introduction}

Nowadays, researchers have developed exciting new materials in nanosize to progress the unique and tunable properties of the applied materials (Sahoo et al. 2007). Due

Renata Dobrucka

renata.dobrucka@ue.poznan.pl

1 Department of Industrial Products Quality and Ecology, Faculty of Commodity Science, Poznan University of Economics, al. Niepodległości 10, 61-875 Poznan, Poland to their small size, nanoparticles exhibit novel material properties, which are significantly different from those of their bulk counterparts (Bindhu et al. 2016). An important aspect of nanoscience is related to the design of experimental methods for the synthesis of nanoparticles (NPs) of different chemical composition, size, shape and properties (Rai and Yadav 2013). Recently, researchers have tried to find biological methods for the synthesis of nanoparticles that will be the alternative to chemical or physical methods. Biological methods for the production of NPs are considered safe and environmentally friendly; they are also costeffective and ensure the complete elimination of toxic chemicals (Okitsu et al. 2007). In addition, the synthesis of NPs using biological means, especially plants, is biocompatible, as they secrete functional biomolecules which actively reduce metal ions (Rai and Ingle 2012).

This study presents a biological method for the synthesis of Mg NPs using the extract of Artemisia abrotanum herb. Magnesium oxide NPs are highly ionic nanoparticulate metal oxides with extremely high surface areas and unusual crystal morphologies (Ravishankar Rai and Jamuna Bai 2011). Nanoscale $\mathrm{MgO}$ possesses unique optical, electronic, magnetic, thermal, mechanical and chemical properties due to its characteristic structures (Ramanujam and Sundrarajan 2014). Magnesium oxide is an important functional metal oxide that has been widely used in various fields, such as catalysis, refractory materials, paints, and superconductors (Salem et al. 2015). In the literature, there are several methods for the synthesis of nano-sized $\mathrm{MgO}$ particles, including the sol-gel method, chemical gas phase deposition, laser vaporization, hydrothermal synthesis, and combustion aerosol synthesis (Mirzaei and Davoodnia 2012). Biological methods for the synthesis of MgO NPs with the use of plant materials have not been widely exploited (Sushma et al. 2015). Based on the literature, 
there are some samples of the synthesis of MgO NPs using Clitoria ternatea, neem leaves (Moorthy et al. 2015), Parthenium (Kumar et al. 2015), Brassica oleracea, Punica granatum peels (Sugirtha et al. 2015), citrus lemon (Awwad et al. 2014) or the extract derived from Nephelium lappaceum L peels (Suresh et al. 2014). Therefore, the application of green synthesis in order to obtain MgO NPs is still an unexplored area, which presents numerous research opportunities.

In this work, the synthesis of magnesium oxide was performed using the extract derived from A. abrotanum herb. The genus of Artemisia (Artemisia L.) includes subshrubs, perennials, plants found mainly in the Holarctic ecozone, on the steppe, on the desert and in the semi-arid areas of Eurasia, Central America and North America. As regards the number of species, this is one of the most numerous genera of the Asteraceae family. A. abrotanum L. has been used in traditional medicine for treating a variety of disorders, including upper airway diseases (Gruenwald 2000). Due to the fact that A. abrotanum $L$. contains significant amounts of polyphenols, together with an original distribution of polyphenols, flavonoids (aglycones and glycosylates) and hydroxycinnamic derivatives, there is an opportunity for new applications of this herbal extract. So far, the literature has not described the synthesis of MgO NPs using A. abrotanum herb extract (Baiceanu et al. 2015). Therefore, the aim of this work was first to obtain and characterize MgO NPs, and then to examine their catalytic activity.

\section{Experimental}

All reagents used in the present work were purchased from Sigma-Aldrich (Poland). Milli-Q water was used throughout the experiment.

\subsection{Synthesis of MgO Nanoparticles}

$\mathrm{MgO}$ NPs were synthesized using clean, dried and powdered A. abrotanum herb. A. abrotanum herb was collected from the region of Silesian Plain (Poland). The powdered A. abrotanum herb was mixed with double distilled water in the proportion: 2 [\% weight] : 98 [\% weight]. The mix was kept at $80{ }^{\circ} \mathrm{C}$ for $40 \mathrm{~min}$ with vigorous magnetic stirring. The obtained solution was filtered through Whatman's No. 1 filter paper. In the next stage, the prepared extract was mixed with $\mathrm{Mg}\left(\mathrm{NO}_{3}\right)_{2}$ in the proportion: 90 [\% weight]: 10 [\% weight] and it was vigorously stirred for $6 \mathrm{~h}$ at $90{ }^{\circ} \mathrm{C}$. The prepared solution was set aside for $24 \mathrm{~h}$ and kept at $25{ }^{\circ} \mathrm{C}$.

\subsection{Characterization of MgO Nanoparticles}

The synthesis of MgO NPs using the extract of A. abrotanum herba was monitored after precipitate formation using UV-Visible spectrophotometer, Cary E 500, at a wavelength range $250-800 \mathrm{~nm}$. The morphology of $\mathrm{MgO}$ NPs was examined by means of scanning electron microscopy (SU3500, Hitachi, with spectral imaging system Thermo Scientific NSS (EDS), the tape of detector (BSE$3 \mathrm{D})$, acceleration voltage $(15.0 \mathrm{kV})$, working distance $(11.6 \mathrm{~mm})$, the pressure (in the case of a variable vacuum conditions) (40 Pa). The functional groups attached to the surface of nanoparticles and the other surface chemical residues were detected using FTIR. The characterization involved Fourier transform infrared spectroscopy (FTIR) analysis of the synthesized MgO NPs by Perkin Elmer Spectrum 1000 spectrum in attenuated total reflection mode, and using the spectral range $4000-400 \mathrm{~cm}^{-1}$ with the resolution of $4 \mathrm{~cm}^{-1}$. The size and structure of $\mathrm{MgO}$ NPs were characterized using a transmission electron microscope JEOL JEM 1200 EXII, operating at $200 \mathrm{kV}$. $\mathrm{X}$-ray diffraction studies of the $\mathrm{MgO}$ NPs were carried out using a BRUKER D8 ADVANCE brand *-2* configuration (generator-detector) X-ray tube copper $S=1.54$ A and LYNXEYE PDS detector. The size of particles was estimated on the basis of Scherrer's formula.

\subsection{Catalytic Properties of MgO Nanoparticles}

The catalytic activity of $\mathrm{MgO}$ NPs synthesized using the extract of A. abrotanum herb was evaluated using UV-Vis spectrophotometer, Cary E 500, to monitor the absorbance peaks. The absorbance was measured in the range $350-800 \mathrm{~nm}$ at room temperature. The proper concentration of methyl orange $\left(1 \times 10^{-4} \mathrm{M}\right)$ was chosen on the basis of the literature review. In the first reaction mixture, the aqueous solution of methyl orange $\left(1 \times 10^{-4} \mathrm{M}\right)$ was monitored by measuring the intensity of absorbance. In the second reaction mixture, $4 \mathrm{ml}$ of methyl orange (MO), $0.5 \mathrm{ml}$ of A. abrotanum water extract and $3 \mathrm{ml}$ of Milli Q water were analyzed. The study of decomposition of methyl orange (MO) used the same amounts of methyl orange and $A$. abrotanum water extract, i.e. $4 \mathrm{ml}$ of $\mathrm{MO}$ and $0.5 \mathrm{ml}$ of $A$. abrotanum water extract. For the variant MO I, $0.5 \mathrm{ml}$ of the prepared solution of MgO NPs and $2.5 \mathrm{ml}$ of Milli $\mathrm{Q}$ water were used. For other variants, i.e. from variant MO II to variant MO V, the amounts of the prepared solution of MgO NPs were successively increased by $0.5 \mathrm{ml}$, and the amounts of Milli $\mathrm{Q}$ water were successively decreased by $0.5 \mathrm{ml}$. All variants were exposed to sunlight for $120 \mathrm{~min}$. 


\subsection{Antioxidant Activity}

In this work, the antioxidant activity was determined by assessing the ability of MgO NPs synthesized using the extract of $A$. abrotanum to neutralize the DPPH (2,2diphenyl-1-picrylhydrazyl) radical, which was reflected by the reduction of the absorbance of the DPPH methanol solution during the reaction with the tested solution of MgO NPs. There were numerous dilutions prepared in the range $0.01-5 \mu \mathrm{g} / \mathrm{ml}$. Changes in the absorbance intensity were measured using the spectrophotometer, Cary E 500. To the test tubes (protected from light) were added $0.1 \mathrm{ml}$ of the tested solution and $0.7 \mathrm{ml}$ of the DPPH reagent at the concentration of $0.1 \mathrm{mM}$. The DPPH reagent was prepared $24 \mathrm{~h}$ in advance (protected from light). After 30 min of shaking the solutions in test tubes, the absorbance was read, at the wavelength $\lambda=515 \mathrm{~nm}$. Water $(0.1 \mathrm{ml})$ and methanol $(0.7 \mathrm{ml})$ were used as reference. Before the measurement of the absorbance of samples, the absorbance of DPPH solution was measured. The measurement was made by measuring the absorbance of $0.1 \mathrm{ml}$ of deionized water and $0.7 \mathrm{ml}$ of DPPH solution. The ability to reduce free DPPH radicals was calculated based on the formula:

$\mathrm{Aa}=(\mathrm{Ao}-\mathrm{Ai} / \mathrm{Ao}) \times 100 \%$,

where Aa means antioxidant activity [\%], Ai means average absorbance of the tested solution, and Ao means average absorbance of the DPPH solution.

\subsection{Statistical Analysis}

The results presented in the work constitute the arithmetic mean of five measurements carried out in parallel. The mean and the standard deviations were calculated using the Statistica 8.0 computer program.

\section{Results and Discussion}

\subsection{UV-Visible Absorption}

UV-Vis absorption spectroscopy is the most widely used method for characterizing the optical properties and electronic structure of nanoparticles, as the absorption bands are relatedto the diameter and aspect ratio of metal nanoparticles (Philip 2008). In this study, the prepared $\mathrm{MgO}$ NPs were confirmed by UV-Vis spectroscopy. According to Feldheim and Foss (2002), light wavelengths in the $300-800 \mathrm{~nm}$ are used for characterizing various metal nanoparticles in the size range $2-100 \mathrm{~nm}$. This work used the range $250-800 \mathrm{~nm}$ to identify $\mathrm{MgO}$ NPs. The prepared solution was set aside for $24 \mathrm{~h}$ and kept at $25^{\circ} \mathrm{C}$.
Figure 1 presents the UV-Visible spectra of MgO NPs synthesized using the extract of $A$. abrotanum herb after $24 \mathrm{~h}$. The absorption spectra of the reaction media with absorbance at $300 \mathrm{~nm}$ confirmed the presence of $\mathrm{MgO}$ NPs. This proves the reduction of $\mathrm{Mg}\left(\mathrm{NO}_{3}\right)_{2}$ and the emergence of $\mathrm{MgO}$. The presence of $\mathrm{MgO}$ was confirmed by conducting such studies as SEM with EDS profiles, TEM, FTIR and DLS. Figure 2 presents the synthesis scheme of $\mathrm{MgO}$ NPs synthesized using of A. abrotanum herba extract.

\subsection{FTIR Analysis}

Fourier transform infrared spectroscopy (FTIR) was used to identify the possible biomolecules which are responsible for the reduction and capping of MgO NPs. Figure 3 presents the FTIR spectra of MgO NPs synthesized using the A. abrotanum herb extract. The spectra show bands at 3308, 2139, 1635, 1346, 419 and $407 \mathrm{~cm}^{-1}$. The strong infrared band near $3308 \mathrm{~cm}^{-1}$ was observed for the $\mathrm{O}-\mathrm{H}$ bond vibrations of hydroxy group. The most intense band at $1635 \mathrm{~cm}^{-1}$ represents vibrations $\mathrm{C}=\mathrm{O}$, typical for the structure of flavonoids which can be found in the $A$. abrotanum herb extract. The peak which appeared at $2139 \mathrm{~cm}^{-1}$ may indicate the presence of alkynes group. The absorption band at $1346 \mathrm{~cm}^{-1}$ is related to $\mathrm{C}-\mathrm{H}$ bending vibrations of aromatic tertiary amine group. The peaks observed at $419 \mathrm{~cm}^{-1}$ and $407 \mathrm{~cm}^{-1}$ indicate the presence of $\mathrm{MgO}$ NPs. FTIR spectrum confirmed the presence of bioactive compounds in A. abrotanum herb. These bioactive compounds were presumed to act as

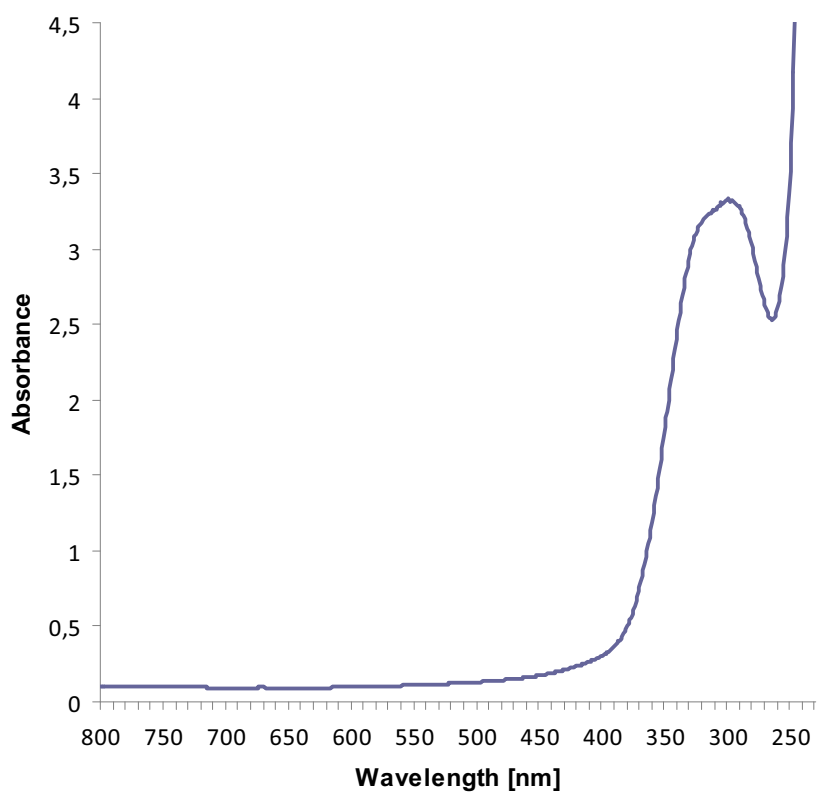

Fig. 1 Absorption spectra of $\mathrm{MgO}$ nanoparticles synthesized using of A. abrotanum herba extract 


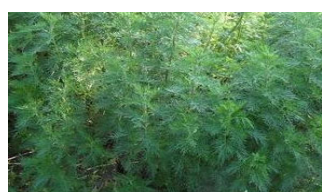

Artemisia abrotanum herba
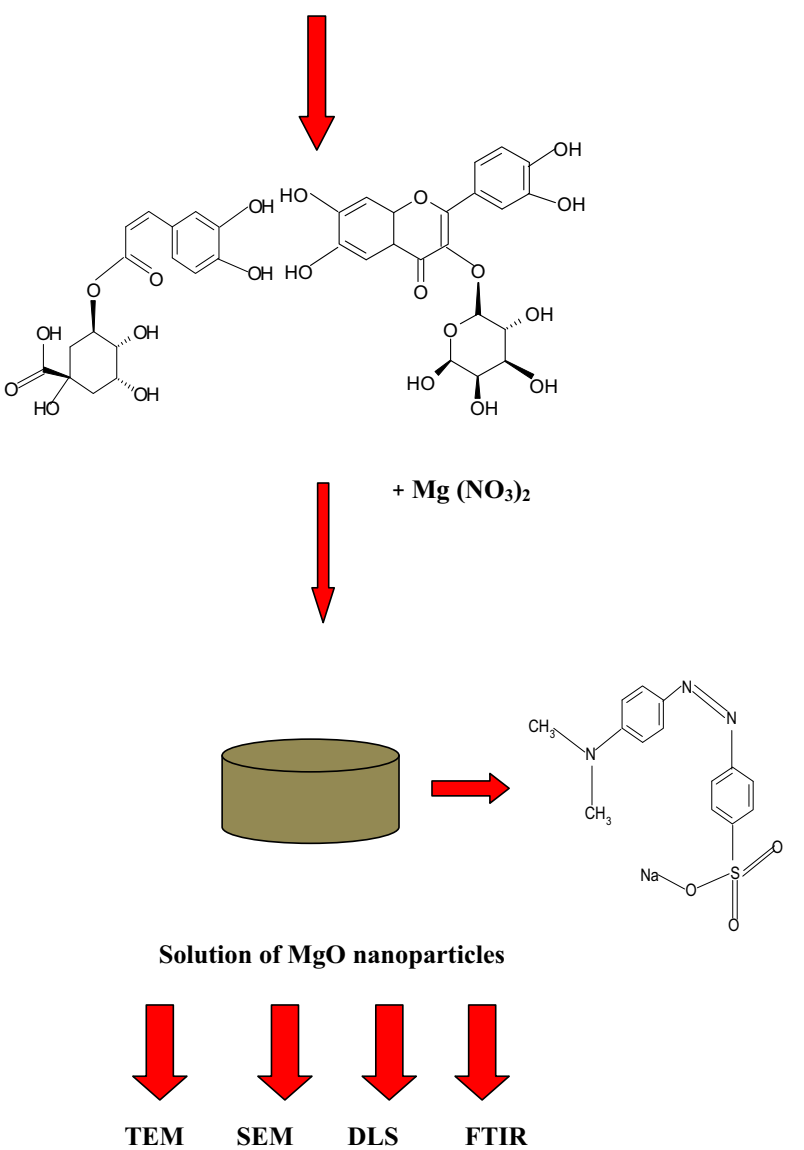

Fig. 2 The scheme synthesis of $\mathrm{MgO}$ nanoparticles synthesized using A. abrotanum herba extract

reducing and capping agents for MgO NPs. According to the literature, the essential oil from A. abrotanum contains 68 identified compounds (Kowalski et al. 2007). The main constituents of the oil are: piperitone, davanone, linanool, 1,8-cineole, silphiperfol-5-en-3-ol $\mathrm{A}$ and germacrene D. Furthermore, in the herbal extract of A. abrotanum L., several phenolic acids were identified: gentisic, caffeic, chlorogenic, $p$ coumaric, ferulic and sinapic acid, both before and after acid hydrolysis. Moreover, A. abrotanum contains several flavonoid aglycones: quercetrol, luteolin, apigenin, and the most abundant flavonoids: hyperoside, rutin, quercetol, patuletin, luteolin, kaempferol. As it is known, phenolic compounds have good antioxidant properties; they inhibit the production of reactive oxygen species (ROS), and thus constitute a protection against oxidative stress. In numerous scientific studies on the antioxidant properties of phenolic acids, researchers have proven the correlation between these properties and the chemical structure. More specifically, the antioxidant properties are related to the number of hydroxy groups in the particle, as well as the level of their esterification. In compounds with one hydroxy group, the antioxidant activity is additionally increased by the presence of one or two methoxy groups in the ring. The introduction of a group with electron donors, alkyl or methoxy, in the orthoposition, reinforces the stability of the antioxidant properties of phenolic acids. (Cuvelier et al. 1996; Shahidi and Wanasundara 1992). The extract of $A$. abrotanum includes chlorogenic acid, which demonstrates high antioxidant activity. The presence of chlorogenic acid and these biologically active compounds is crucial for the reduction and stabilization of MgO NPs. Figure 4 presents the structure of the selected phenolic acids (chlorogenic and caffeic acid) and flavonoids (hyperoside, kaempferol).

\subsection{XRD Analysis}

The XRD techniques are widely used to determine the size of particles and the structure of nanoparticles. Figure 5 shows the X-ray diffraction profile of MgO NPs synthesized using the A. abrotanum herb extract. The size of MgO NPs was obtained by Debye-Scherrer's formula:

$D=\mathrm{K} \lambda /(\beta \cos \theta)$

where $D$ is the crystal size; $\lambda$ is the wavelength of the $\mathrm{X}$-ray radiation $(\lambda=0.15406 \mathrm{~nm})$ for $\mathrm{CuK} \alpha ; K$ is usually taken as 0.9 ; and $\beta$ is the line width at half-maximum height.

$\mathrm{MgO}$ NPs show peaks corresponding to the planes at $2 \theta=39.60^{\circ}(111), 2 \theta=42.90^{\circ}$ (200), $2 \theta=62.86^{\circ}$ (202), $2 \theta=74.62^{\circ}(311), 2 \theta=78.60^{\circ}(222)$. The XRD spectrum suggests that $\mathrm{MgO}$ NPs synthesized using the A. abrotanum herb extract were crystalline. The crystal sizes calculated using Scherrer's formula were about $10 \mathrm{~nm}$.

\subsection{SEM and EDS Profile}

Figures 6 present the scanning electron microscopy (SEM) images of the MgO NPs synthesized using the A. abrotanum herb extract where the scale bar is (A) $5 \mu \mathrm{m}$, (B) $10 \mu \mathrm{m}$, (C) $20 \mu \mathrm{m}$ and (D) $30 \mu \mathrm{m}$. Figure 6 shows that the size of single MgO NPs was about $10 \mathrm{~nm}$. Furthermore, the MgO NPs were well dispersed. Locally, the synthesized $\mathrm{MgO}$ NPs were agglomerated and formed larger clusters. Figure 7 presents the SEM images of the MgO nanoparticles synthesized using the $A$. abrotanum herb extract where (A) the scale bar is $25 \mu \mathrm{m}$ and (C) the scale bar is $10 \mu \mathrm{m}$ with EDS profiles. Further analysis of the MgO NPs by EDS profile confirmed the signal characteristic of 


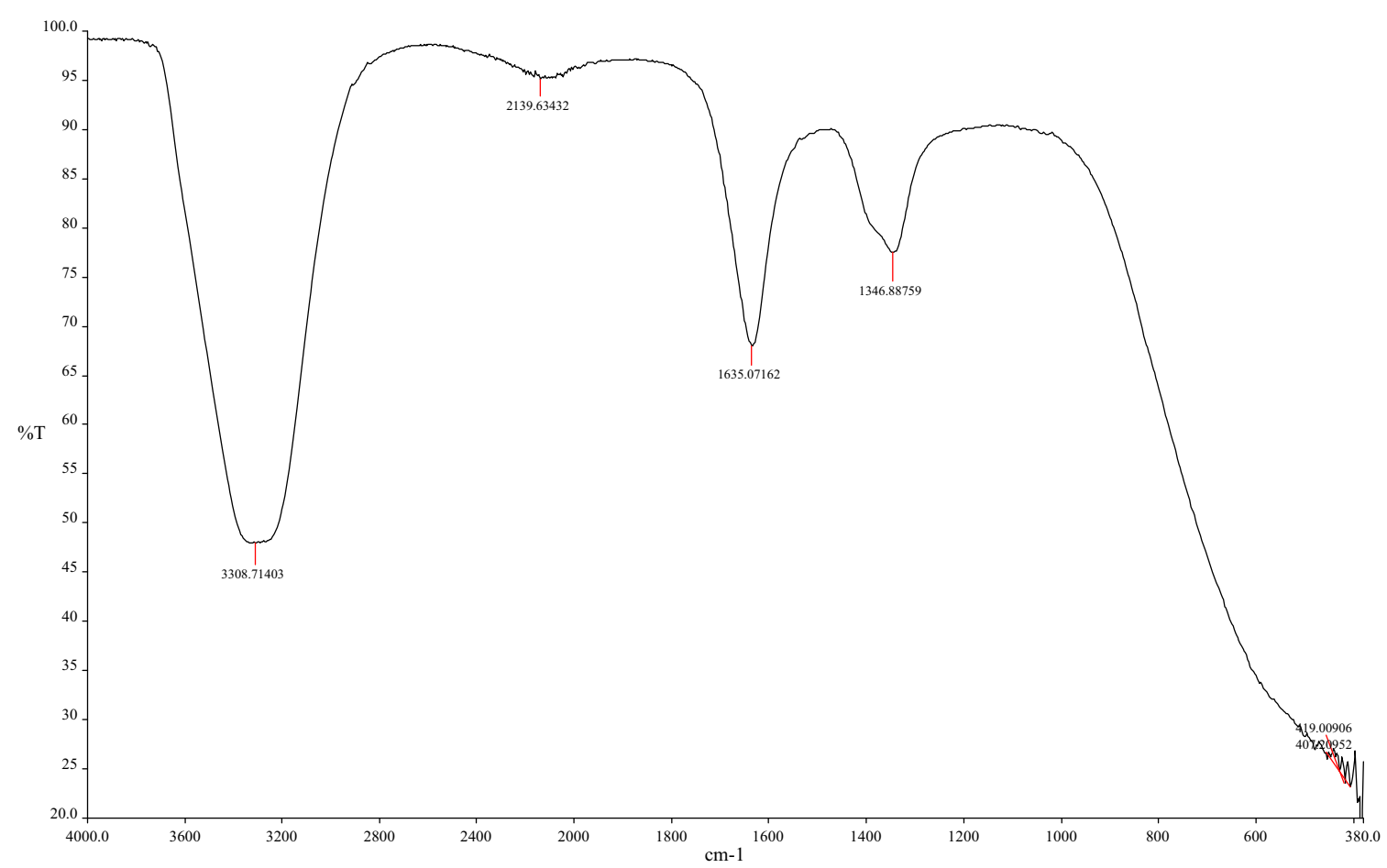

Fig. 3 FTIR spectra of $\mathrm{MgO}$ nanoparticles synthesized using A. abrotanum herba extract

Fig. 4 The structure of selected phenolic acids: a chlorogenic, b caffeic acid and flavonoids, c hyperoside, $\mathbf{d}$ kaempferol<smiles>O=C(/C=C\c1ccc(O)c(O)c1)O[C@@H]1C[C@](O)(C(=O)O)C[C@H](O)[C@H]1O</smiles>

(A)<smiles>O=C1C(OC2O[C@H](O)C(O)[C@H](O)[C@H]2O)=C(c2ccc(O)c(O)c2)OC2C=C(O)C(O)=CC12</smiles>

(C)<smiles>O=C(O)/C=C/c1ccc(O)c(O)c1</smiles>

(B)<smiles>O=c1c(O)c(-c2ccc(O)cc2)oc2cc(O)cc(O)c12</smiles>

(D) 


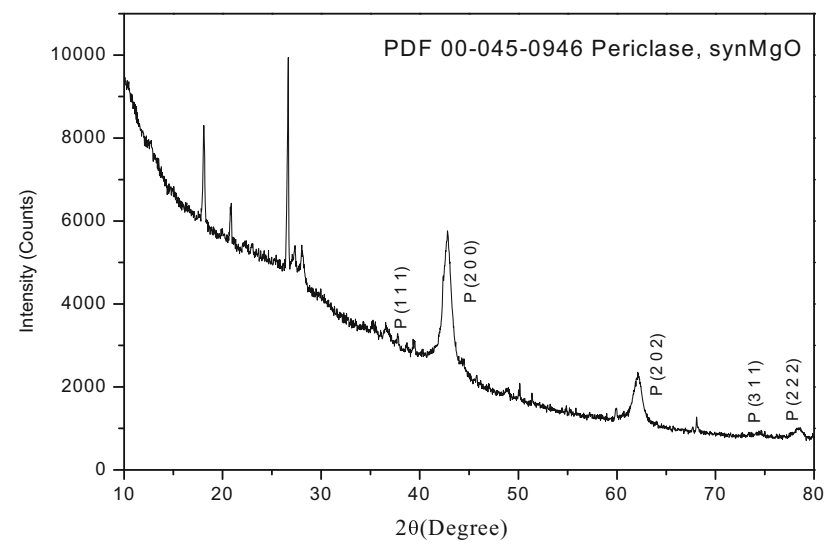

Fig. 5 XRD patterns of synthesized $\mathrm{MgO}$ nanoparticles using $A$. abrotanum herba extract

magnesium and oxygen. EDS profile is the additional evidence of the formation of pure $\mathrm{MgO}$ nanoparticles. Figure $7 \mathrm{~b}, \mathrm{~d}$ presents peaks between 0.5 and $1.5 \mathrm{kV}$, which indicate the presence of MgO NPs. Figure 7 (B) shows the magnesium content at the level of $25.4 \%$, and the oxygen content at the level of $47.6 \%$. The quantities of the remaining elements are as follows: $\mathrm{C}(23.7 \%), \mathrm{Al}(0.7 \%)$, $\mathrm{Si}(0.3 \%), \mathrm{P}(0.1 \%), \mathrm{K}(1.0 \%)$ and $\mathrm{Ca}(0.5 \%)$. Figure 7 (D) shows the magnesium content at the level of $13.9 \%$, and the oxygen content at the level of $39.4 \%$. The quantities of the remaining elements are as follows: $\mathrm{C}(43.8 \%)$, $\mathrm{Al}(1.4 \%), \mathrm{Si}(0.3 \%), \mathrm{K}(0.8 \%)$ and $\mathrm{Ca}(0.5 \%)$.

The size, shape, and morphologies of the synthesized $\mathrm{MgO}$ nanoparticles were examined using transmission electron microscopy (TEM).

\subsection{TEM Analysis}

The size of the synthesized MgO NPs was characterized by using transmission electron microscopy (TEM). As it is known, TEM is an extremely useful technique to obtain the direct information concerning particle size distribution, mean particle size and the shape of nanoparticles. Transmission electron microscopy has a 1000 times higher resolution than does scanning electron microscopy (Eppler et al. 2000). In this study, it was decided to use both methods in order to measure the size of nanoparticles. The TEM image (Fig. 8 A magnification 50,000 $\times$ and Figure B magnification $100,000 \times$ ) confirmed that the size of the synthesized MgO NPs was less than $10 \mathrm{~nm}$. Moreover, the transmission electron microscopy image presents the spherical structure of the synthesized $\mathrm{MgO}$ NPs.
Fig. 6 SEM images of the synthesized $\mathrm{MgO}$ nanoparticles synthesized using A. abrotanum herba extract where the scale bar is a $5 \mu \mathrm{m}$, b $10 \mu \mathrm{m}$, c $20 \mu \mathrm{m}$ and d $30 \mu \mathrm{m}$
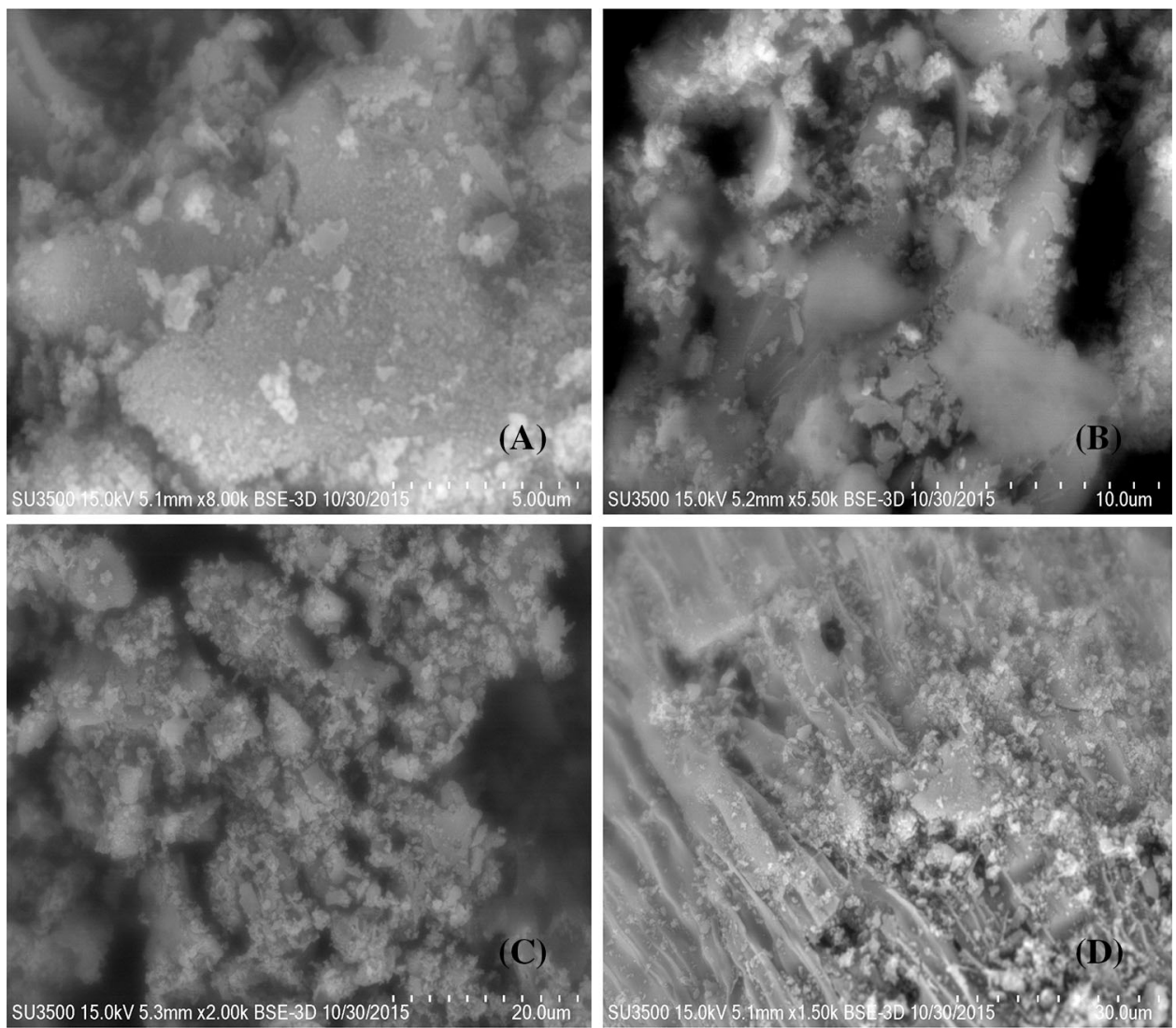

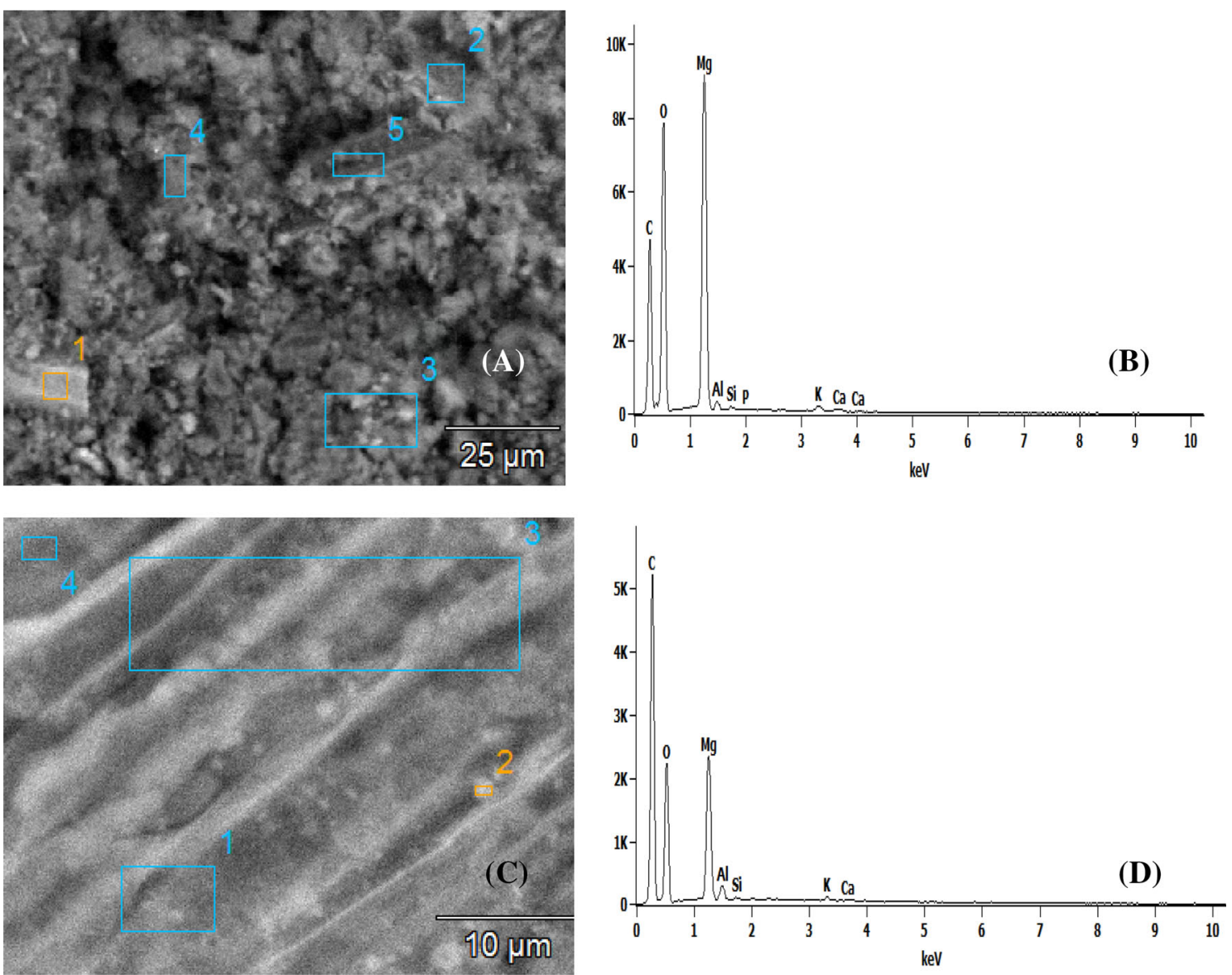

Fig. 7 SEM images of the MgO nanoparticles where a the scale bar is $25 \mu \mathrm{m}$ and $\mathbf{c}$ the scale bar is $10 \mu \mathrm{m}$ and EDS profiles (b, d)
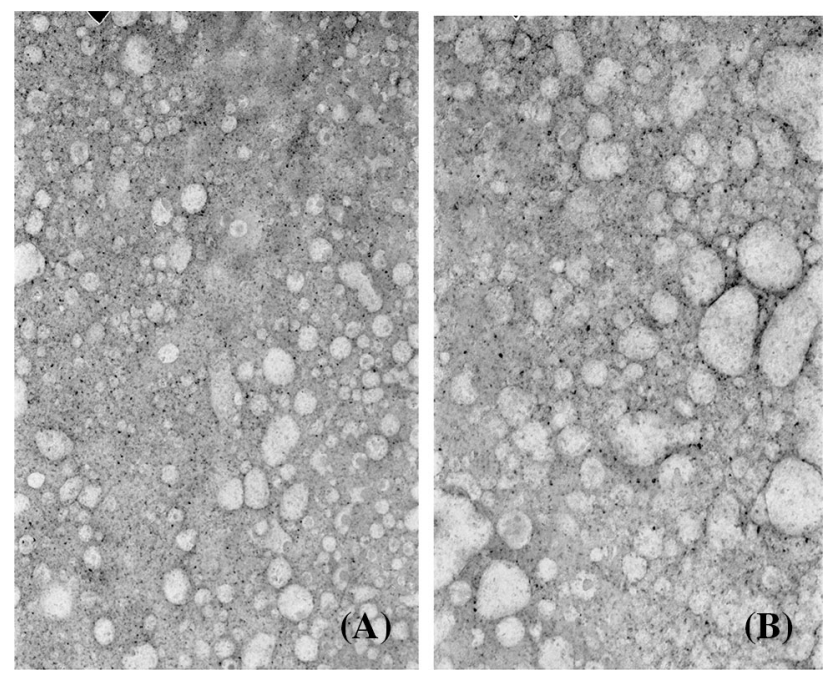

Fig. 8 TEM image of the synthesized $\mathrm{MgO}$ nanoparticles a magnification $50,000 \times$ and $\mathbf{b}$ magnification $100,000 \times$

\subsection{Catalytic Activity of MgO Nanoparticles}

Organic dyes are widely used in many industries. Methyl orange (MO) is a dye which is widely applied in the chemical, textile and paper industries. As the excessive use of organic dyes leads to environmental pollution, the control of industrial effluents is an indispensable job, which helps in the creation of harmless and clean environment (Ganapuram et al. 2015). Therefore, the search for a simple method for the efficient degradation of dyes has gained greater significance. One of such solutions may be the application of metal oxide nanoparticles, which show enhanced catalytic activity in the degradation of organic dyes (Ashokkumar et al. 2014). In this study, MgO NPs were used as a catalyst for the remediation of methyl orange (MO). Figure 9 shows the reduction of methyl orange (MO) in the absence of synthesized MgO NPs for a time period of $120 \mathrm{~min}$. The study used variants from MO I to $\mathrm{MO} \mathrm{V}$; in each variant, the amount of the prepared solution of $\mathrm{MgO}$ NPs was successively increased by $0.5 \mathrm{ml}$, while the amount of water was successively decreased by $0.5 \mathrm{ml}$. The maximum absorbance wavelength of methyl orange was recorded at $464 \mathrm{~nm}$. The diagram explicitly shows that the degradation of $\mathrm{MO}$ advances due to the increasing amount of the prepared solution of MgO NPs. This study confirms the catalytic properties of green synthesized $\mathrm{MgO}$ NPs. These results 
Fig. 9 Absorption spectra of methyl orange reduction by $\mathrm{MgO}$ nanoparticles synthesized using A. abrotanum herba extract

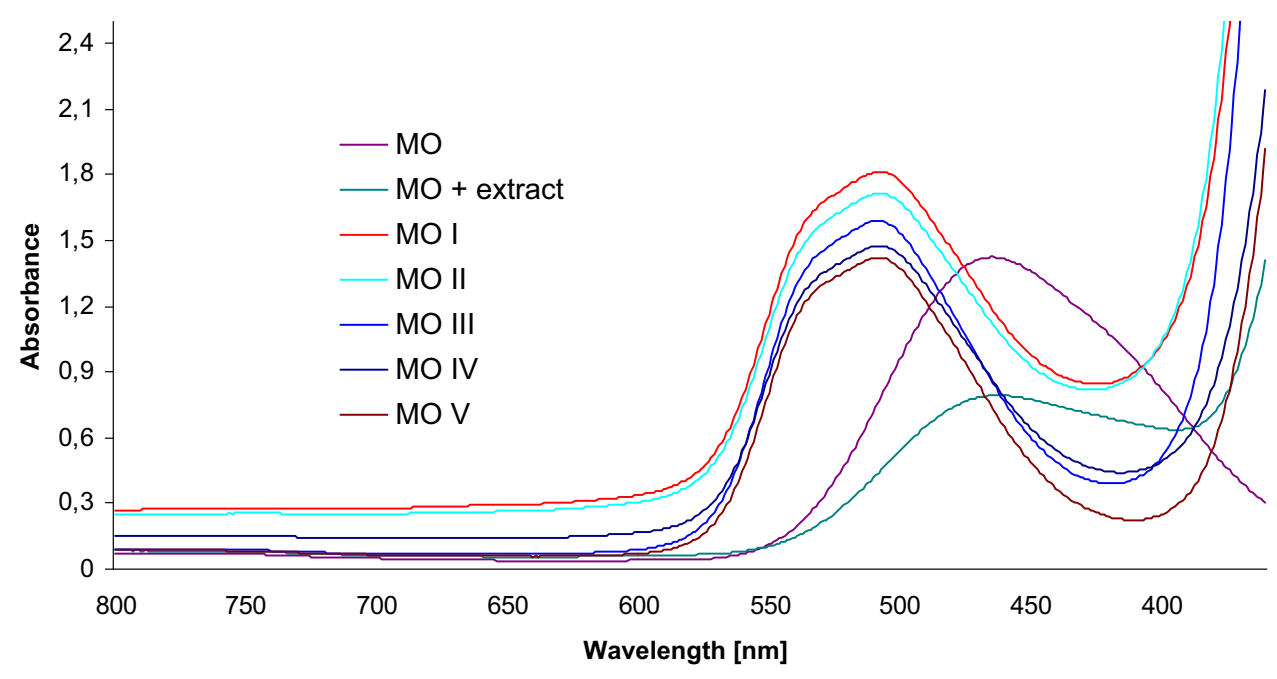

may be complementary to the studies conducted by Gupta et al. (2011), who synthesized gold, silver and platinum nanoparticles using tannic acid as the reducing agent. The prepared metal nanoparticles were used for the catalytic degradation of methyl orange in the presence of $\mathrm{NaBH}_{4}$. The international literature describes only few studies related to the catalytic properties of the MgO NPs obtained as a result of biological synthesis. One of such studies is presented by Mirzaei and Davoodnia (2012), who used microwave-assisted sol-gel method for the preparation of nano-sized $\mathrm{MgO}$ particles. The synthesized $\mathrm{MgO}$ NPs exhibited high catalytic activity and gave the desired products in good to high yields. This work presents the catalytic activity of biologically synthesized MgO NPs, which may be applied in the degradation of organic dyes.

\subsection{Antioxidant Activity of MgO Nanoparticles}

The antioxidant activity of MgO NPs synthesized using the A. abrotanum herb extract was determined by means of the test using the DPPH radical. In the DPPH method, antioxidants present in the tested sample reduce the stable nitrogen radical 2,2-diphenyl-1-picrylhydrazyl (DPPH), causing the decrease in absorbance measured at the wavelength of $515 \mathrm{~nm}$. Substances that can donate an oxygen atom create the reduced form of DPPH; in consequence, the solution loses its violet color. In this work, the solution of the active radical was purple, which proved that the previously unpaired electron became paired. The level of color change of the DPPH solution after adding the solution with antioxidants shows the free radical scavenging properties of the antioxidants. This study also investigated the antioxidant activity of the only extract of $A$. abrotanum herb. There were five separate measurements conducted, which were repeated five times. In this work, the antioxidant properties of MgO NPs synthesized using the A. abrotanum herb extract were determined by calculating the IC50 parameter. This parameter determines the ability to scavenge free radicals. The higher the IC50 parameter, the more reactive the antioxidant. For $\mathrm{MgO}$ NPs, the mean value of the parameter, based on five measurements, was $4.73 \mu \mathrm{g} / \mathrm{ml}$. For comparison, for the extract of $A$. abrotanum herb, the mean value of the parameter, based on five measurements, was $6.28 \mu \mathrm{g} / \mathrm{ml}$. The obtained result indicated the high antioxidant activity of the obtained $\mathrm{MgO}$ NPs. The presence of $\mathrm{MgO}$ nanoparticles led to a considerable improvement of the antioxidant activity of the $A$. abrotanum herb extract. Table 1 shows the results obtained for the antioxidant activity of $\mathrm{MgO}$ NPs synthesized using the A. abrotanum herb extract, as well as of the A. abrotanum herb extract itself.

\section{Conclusion}

Nanoscience and technology have become the new hot topics in material science. Nanostructured oxide materials have been studied extensively because of their large surface areas, unusual adsorptive properties, surface defects and fast diffusivities. Recently researchers have discovered the possibilities of preparing nanomaterials in an aqueous medium with the help of stabilizing, capping or hydrolytic

Table 1 The results of antioxidant activity of $\mathrm{MgO}$ NPs and the extract of A. abrotanum herb

\begin{tabular}{lll}
\hline Product & $\mathrm{IC}_{50}(\mu \mathrm{g} / \mathrm{ml})$ & $\mathrm{SD}$ \\
\hline MgO NPs & 4.73 & 0.10 \\
A. abrotanum herb extract & 6.28 & 0.01 \\
\hline
\end{tabular}

IC50 inhibitory concentration $50 \%, S D$ standard deviation 
agents. This study also examined the roles of the water extract of A. abrotanum herb in the formation and stabilization of MgO NPs. The synthesized nanostructures have been characterized by UV-Vis, FTIR, XRD, TEM and SEM with EDS profile. The obtained MgO NPs, sized about $10 \mathrm{~nm}$, have shown the catalytic activity in the photodegradation of MO. To sum up, this study presents the ecological method for preparing nanoparticles without the need of using harmful chemical substances. The obtained MgO NPs may have potential applications due to their good catalytic activity. MgO NPs also showed strong antioxidant properties.

Acknowledgments Research on the synthesis of $\mathrm{MgO}$ nanoparticles using A. abrotanum herba extracts and their catalytic activity has been financed from the grant for young researchers in 2015 by the Ministry of Science and Higher Education.

Open Access This article is distributed under the terms of the Creative Commons Attribution 4.0 International License (http://crea tivecommons.org/licenses/by/4.0/), which permits unrestricted use, distribution, and reproduction in any medium, provided you give appropriate credit to the original author(s) and the source, provide a link to the Creative Commons license, and indicate if changes were made.

\section{References}

Ashokkumar S, Ravi S, Kathiravan V, Velmurugan S (2014) Synthesis, characterization and catalytic activity of silver nanoparticles using Tribulus terrestris leaf extract, Spectrochim. Acta Part A Mol Biomol Spectrosc 121:88-93

Awwad AM, Ahmad AL (2014) Biosynthesis, characterization, and optical properties of magnesium hydroxide and oxide nanoflakes using Citrus limon leaf extract. Arab J Phys Chem 1(2):66

Baiceanu E, Vlase L, Baiceanu A, Nanes M, Rusu D, Crisan G (2015) New polyphenols identified in artemisiae abrotani herba extract. Molecules 20:11063-11075

Bindhu MR, Umadevi M, Micheal MK, Arasu MV, Al-Dhabi NA (2016) Structural, morphological and optical properties of $\mathrm{MgO}$ nanoparticles for antibacterial applications. Mater Lett 166:19-22

Cuvelier ME, Richard H, Berset C (1996) Antioxidative activity and phenolic composition of pilot-plant and commercial extracts of sage and rosemary. J Am Oil Chem Soc 73(5):645-652

Eppler AS, Zhu J, Anderson EA, Somorjai GA (2000) Model catalysts fabricated by electron beam lithography: AFM and TPD surface studies and hydrogenation/dehydrogenation of cyclohexene $+\mathrm{H} 2$ on a Pt nanoparticle array supported by silica. Top Catal 13:33-41

Feldheim DL,. Foss CA (eds) (2002) Metal nanoparticles: synthesis, characterization and applications (Marcel Dekker Inc.)

Gruenwald J (2000) PDR for herbal medicines. Montvale

Gupta N, Singh HP, Kumar Dharma R (2011) Metal nanoparticles with high catalytic activity in degradation of methyl orange: an electron relay effect. J Mol Catal A: Chem 335:248-252
Kowalski R, Wawrzykowski J, Zawiślak G (2007) Analysis of essential oils and extracts from Artemisia abrotanum L. and Artemisia dracunculus L. Herba Pol 53(3):246-254

Kumar D, Reddy Yadav LS, Lingaraju K, Manjunath K, Suresh D, Prasad D, Nagabhushana H, Sharma SC, Raja Naika H, Chikkahanumantharayappa, Nagaraju G (2015) Combustion synthesis of $\mathrm{MgO}$ nanoparticles using plant extract: structural characterization and photoluminescence studies. AIP Conf Proc 1665:050145

Mirzaei H, Davoodnia A (2012) Microwave assisted sol-gel synthesis of $\mathrm{MgO}$ nanoparticles and their catalytic activity in the synthesis of hantzsch 1, 4-dihydropyridines. Chin J Catal 33:1502-1507

Moorthy SK, Ashok CH, Venkateswara Rao K, Viswanathan C (2015) Synthesis and characterization of $\mathrm{MgO}$ nanoparticles by Neem leaves through green method. Mater Today Proc 2:4360-4368

Okitsu K, Mizukoshi Y, Yamamoto TA, Maeda Y, Nagata Y (2007) Sonochemical synthesis of gold nanoparticles on chitosa. Lett Mater 61(16):3429-3431

Philip D (2008) Synthesis and spectroscopic characterization of gold nanoparticles. Spectrochimica Acta Part A 71:80-85

Rai M, Ingle A (2012) Role of nanotechnology in agriculture with special reference to management of insect pests 94(2):287-293

Rai M, Yadav A (2013) Plants as potential synthesizer of precious metal nanoparticles: progress and prospects. IET Nanobiotechnol 3:117-124

Ramanujam K, Sundrarajan M (2014) Antibacterial effects of biosynthesized $\mathrm{MgO}$ nanoparticles using ethanolic fruit extract of Emblica officinalis. J Photochem Photobiol, B 141:296-300

Ravishankar RV, Jamuna BA (2011) Nanoparticles and their potential application as antimicrobials. Science against microbial pathogens. Communicating current research and technological advances. A. Méndez-Vilas (Ed.), pp 197-209

Reddy Ganapuram B, Alle M, Dadigala R, Dasari A, Maragoni V, Guttena V (2015) Catalytic reduction of methylene blue and Congo red dyes using green synthesized gold nanoparticles capped by salmalia malabarica gum. Int Nano Lett 5:215-222

Sahoo SK, Parveen S, Panda JJ (2007) The present and future of nanotechnology in human health care. Nanomed NBM 3(1):20-31

Salem JK, El-Nahhal IM, Hammad TM, Kuhn S, Sharekh SA, ElAskalani M, Hempelmann R (2015) Optical and fluorescence properties of $\mathrm{MgO}$ nanoparticles in micellar solution of hydroxyethyl laurdimonium chloride. Chem Phys Lett 636:26-30

Shahidi F, Wanasundara JPD (1992) Phenolic antioxidant. Crit Rev Food Sci Nutr 32:67-103

Sugirtha P, Divya R, Yedhukrishnan R, Suganthi KS, Anusha N, Ponnusami V, Rajan KS (2015) Green synthesis of magnesium oxide nanoparticles using brassica oleracea and punica granatum peels and their anticancer and photocatalytic activity. Asian J Chem 27(7):2513-2517

Suresh J, Yuvakkumar R, Sundrarajan M, Hong SI (2014) Green synthesis of magnesium oxide nanoparticles. Adv Mater Res 952:141-144

Sushma NJ, Prathyusha D, Swathi G, Madhavi T, Deva Prasad Raju B, Mallikarjuna K, Hak-Sung Kim (2015) Facile approach to synthesize magnesium oxide nanoparticles by using Clitoria ternatea-characterization and in vitro antioxidant studies. Appl Nanosci 1-8 